\title{
Experimental Test of the Sweet-Parker Model of Magnetic Reconnection
}

\author{
Hantao Ji, Masaaki Yamada, Scott Hsu, and Russell Kulsrud \\ Plasma Physics Laboratory, Princeton University, P.O. Box 451, Princeton, NJ 08543
}

(September 29, 1997)

\begin{abstract}
We report a quantitative experimental test of the Sweet-Parker model of magnetic reconnection in a controlled laboratory plasma. It is found that the observed reconnection rate cannot be explained by the Sweet-Parker model unless the model is generalized to incorporate compressibility, downstream pressure, and the effective resistivity. The latter is significantly enhanced over its classical values in the collisionless limit.
\end{abstract}

PACS numbers: 52.30.Jb, 96.60.Rd, 94.30.Lr

Typeset using REVTEX 
Magnetic reconnection plays a crucial role in determining the topology of magnetic fields of magnetohydrodynamic (MHD) plasmas in space and in the laboratory [1-3]. Although this is a localized process, it often causes fundamental changes in macroscopic configurations, such as in solar flares [4], magnetospheric substorms [4], and relaxation processes in laboratory plasmas [5]. Magnetic reconnection also provides the most plausible mechanism for releasing the energy stored in the magnetic field to plasma kinetic and thermal energies observed in solar flares, auroral phenomena, and laboratory plasmas. Although the concept of magnetic reconnection was first suggested by Giovanelli [6] more than fifty years ago, quantitative models did not exist until proposals by Sweet [7] and Parker [8] appeared ten years later. Since then, however, the validity of the Sweet-Parker model has been questioned because its predicted reconnection rate is too slow to explain explosive solar flares. Instead, the attention has shifted to Petschek's model [9] and other models based on standing shock waves $[10,11]$, which predicted faster reconnection rates. Availability of computer simulation as a research tool has brought about an explosive amount of literature [12] on magnetic reconnection physics in great detail.

Despite the theoretical and computational progress made in past decades on magnetic reconnection, none of these models have been verified or even tested in the laboratory or in space. Stenzel and Gekelman [13] carried out a series of experiments in a linear device and in the electron MHD regime where only electrons are magnetized. Although detailed local fluctuations were measured, quantitative tests of leading theoretical models were not made. More recent experiments have focused on the effects of the third field component during reconnection from both global [14] and local [15] points of view. In this letter, we report a quantitative experimental test of the Sweet-Parker model in the Magnetic Reconnection Experiment (MRX) [15,16], where quasi 2D magnetic reconnection is realized in the MHD regime. A significant finding is that the observed reconnection rate cannot be explained by the Sweet-Parker model unless the model is generalized to include compressibility, downstream pressure, and the effective resistivity. The latter is significantly enhanced over its classical values in the collisionless limit. However, shock structures, which are an essential 
feature of the Petschek-type models, have not been observed within sensitivity limits of the measurements in MRX.

An example of driven magnetic reconnection in MRX is displayed in Fig. 1, where both magnetic field vector $B$ and poloidal flux $\Psi$ contours measured in a single discharge are plotted in a $R-Z$ plane. All results reported here are for the case of anti-parallel reconnection where the toroidal field is negligible. Magnetic reconnection is induced by changing currents in two flux cores whose toroidally symmetric shape ensures the quasi 2D geometry [16]. As the oppositely directed magnetic field lines $\left(B_{Z}\right)$ move in toward each other in the $R$ direction, a sharp sheet current develops perpendicular to the plane of the page. The sheet current diffuses due to plasma resistivity in this "diffusion region," where a magnetic field line can lose its original identity and reconnect to another field line. The reconnected field lines $\left(B_{R}\right)$ then move away along the $Z$ direction.

The motion of magnetic field lines in an MHD plasma with resistivity $\eta$ is described by

$$
\frac{\partial \boldsymbol{B}}{\partial t}=\nabla \times(\boldsymbol{V} \times \boldsymbol{B})+\frac{\eta}{\mu_{0}} \nabla^{2} \boldsymbol{B}
$$

where $\boldsymbol{V}$ is the flow velocity. The first term on the right hand side represents the effect of plasma convection while the second term describes field line diffusion. Significance of the diffusion term is represented by $1 / S$ where the Lundquist number $S$ is defined by $\mu_{0} L V_{A} / \eta$. Here $V_{A} \equiv B / \sqrt{\mu_{0} \rho}$ ( $\rho=$ mass density) is the Alfvén speed and $L$ is the typical plasma size. For typical MHD plasmas such as solar flares [4], $S>10^{10}$; for tokamaks, $S>10^{7}$; and for MRX plasmas, $S \lesssim 10^{3}$.

Another important equation governing reconnection is the continuity equation,

$$
\frac{\partial n}{\partial t}+\nabla \cdot(n \boldsymbol{V}) \approx \frac{\partial \bar{n}}{\partial t}-\frac{\bar{n} V_{R}}{\delta}+\frac{\bar{n} V_{Z}}{L}=0
$$

where $\delta(L)$ is the thickness (width) of the current sheet and $\bar{n}$ is the averaged density in the diffusion region. Integration of the equation of motion, $\rho(\partial / \partial t+\boldsymbol{V} \cdot \boldsymbol{\nabla}) \boldsymbol{V}=-\boldsymbol{\nabla} p+\boldsymbol{j} \times \boldsymbol{B}$, (i.e., the $R$ component across the current sheet and the $Z$ component along the current sheet) gives 


$$
\rho \frac{\partial V_{R}}{\partial t}+p_{\text {up }}+\frac{B_{Z}^{2}}{2 \mu_{0}}+\frac{1}{2} \rho V_{R}^{2}=p_{0}=\rho \frac{\partial V_{Z}}{\partial t}+p_{\text {down }}+\frac{B_{R}^{2}}{2 \mu_{0}}+\frac{1}{2} \rho V_{Z}^{2},
$$

where $p_{0}, p_{\text {up }}$ and $p_{\text {down }}$ are plasma pressures at the center and in the upstream and downstream regions, respectively. $V_{R}\left(V_{Z}\right)$ is the reconnection speed in the upstream (downstream) region. The Sweet-Parker model [7,8] assumes steady state reconnection $(\partial B / \partial t=0)$ in an incompressible plasma $(\partial n / \partial t=0)$ with uniform pressure outside the diffusion region $\left(p_{\text {up }}=p_{\text {down }}\right)$ and with negligible $\rho V_{R}^{2} / 2$ and $B_{R}^{2} / 2 \mu_{0}$. Then Eqs. (1), (2) and (3) can be rewritten as $V_{R}=\eta / \mu_{0} \delta, V_{R}=(\delta / L) V_{Z}$ and $V_{Z}=V_{A}$, resulting in a simple expression for the reconnection rate as measured by the Alfvén Mach number, $M_{A} \equiv V_{R} / V_{A}=\sqrt{\eta / \mu_{0} L V_{A}}=1 / \sqrt{S}$.

The Sweet-Parker model can be tested if all the basic plasma parameters are adequately measured. The low temperature $(<50 \mathrm{eV})$ and short-pulsed $(<1 \mathrm{~ms})$ MRX plasmas have the advantage that internal probes can be used routinely. Langmuir probes with triple pins measure electron density $\left(n_{e}\right)$ and temperature $\left(T_{e}\right)$ simultaneously. The plasma density measurement has been calibrated by an interferometer which measures the line-integrated density. All three components of $\boldsymbol{B}$ are measured during the reconnection process by a 90 channel 2D pick-up coil array with $4 \mathrm{~cm}$ resolution (as shown in Fig. 1). A finer 1D pick-up probe array with $0.5 \mathrm{~cm}$ resolution is used to measure the $B_{Z}$ profile across the current sheet [15]. The measured $B_{Z}$ profiles are fit into the Harris-type function [17], $\tanh [(R-$ $\left.R_{0}\right) / \delta$, to determine $\delta$ and peak current density. Local flow velocity can be determined either by a Mach probe or time evolution of $\Psi(R, Z)$, i.e., $V_{X}=-(\partial \Psi / \partial t) /(\partial \Psi / \partial X)(X=R$ in the upstream region and $X=Z$ in the downstream region). The latter method is valid when the resistive effects are negligible, a condition satisfied outside the diffusion region. Results by both methods agree well with each other and the latter has been used routinely because of its convenience. Probe perturbation of the plasma is quantitatively estimated and observed to be less than 5\% [16]. Typical plasma parameters are as follows: $B<0.5 \mathrm{kG}$, $T_{e}=10-20 \mathrm{eV}$, and $n_{e}=0.2-1.5 \times 10^{20} \mathrm{~m}^{-3}$.

In general, the Lundquist number $S$ is calculated from the measured $T_{e}$ based on the 
Spitzer resistivity (parallel resistivity, $\left.\eta_{\|}\right)$. However, perpendicular resistivity $\eta_{\perp}\left(=2 \eta_{\|}\right)$ should be used in the case of anti-parallel reconnection since the current flows essentially perpendicular to the field. A more detailed calculation which incorporates profile effects of density and temperature gives a nearly identical expression for resistivity [18].

A shot-averaged time evolution of plasma parameters for driven reconnection is shown in Fig. 2. The current density peaks at $t=290 \mu \mathrm{s}$, when $\delta$ is minimized and reconnection speed $V_{R}$ reaches its steady state of about $3 \mathrm{~km} / \mathrm{s}$. The $n_{e}$ measured at the center of the current sheet keeps increasing until a later time, while $T_{e}$ at the same location remains almost constant at 10-15 eV (not shown). A series of experiments has been performed in which $B_{Z}$ is varied while other conditions are kept constant, including the fill pressure $p_{\text {fill }}$ (6 mTorr). It is observed that the reconnection rate decreases as $B_{Z}$ increases.

A straightforward test of the Sweet-Parker model is shown in Fig. 3(a) where the reconnection rate is plotted against $1 / \sqrt{S}$. Clearly, the observation does not agree with the Sweet-Parker prediction (dotted line). When the reconnection rate changes by a factor of $3,1 / \sqrt{S}$ changes only by a factor of 1.5 . Causes of the discrepancy can be found by systematically examining the validity of Eqs. (1), (2), and (3).

The first equation to be evaluated is Ohm's law in the toroidal direction, $E_{T}+V_{R} \times B_{Z}=$ $\eta_{\perp} j_{T}$, which has been used to derive Eq. (1). All three terms are measured across the current sheet. As shown in the inset of Fig. $4, E_{T}(=-\dot{\Psi} / 2 \pi R)$ balances with $V_{R} \times B_{Z}$ outside the diffusion region and $\eta_{\perp} j_{T}$ inside the diffusion region. In this example, the measured effective resistivity $\left(\eta_{\perp}^{*}=E_{T} / j_{T}\right)$ is about twice its classical value. It is found that the enhancement of resistivity is a strong function of collisionality (characterized by the dimensionless parameter $\left.\lambda_{\mathrm{mfp}} / \delta\right)$, as shown in Fig. 3. A significant enhancement $(\sim 10)$ of the resistivity is observed in the collisionless regime $\left(\lambda_{\mathrm{mfp}} \gg \delta\right)$. We note that electron-neutral collisions are estimated to be negligible compared to Coulomb collisions in the present experimental regimes.

By using $\eta_{\perp}^{*}$ to calculate the Lundquist number (denoted by $S^{*} \equiv \mu_{0} L V_{A} / \eta_{\perp}^{*}$ ), the SweetParker model can be tested again, as plotted in Fig. 3(b). A clear linear dependence can be seen between $M_{A}$ and $1 / \sqrt{S^{*}}$, but the magnitude is off by a factor of two. Equation (1) is 
satisfied when the effective resistivity is used and the steady state assumption $(\partial B / \partial t \approx 0)$ holds. Therefore, the discrepancy must reside in the continuity [Eq. (2)] and/or momentum [Eq. (3)] equations.

The incompressibility assumption does not hold since the $\partial n / \partial t$ term is not negligible compared to the other terms in the continuity equation as seen in Fig. 2, which shows increasing density in the current sheet during reconnection. Retaining this term in the continuity equation leads to an increased inflow, $V_{R}=(\delta / L)\left(V_{Z}+L \dot{n} / n\right)$, due to an accumulation of density at the center. We note that the particle source term in the diffusion region is negligible in the present experimental regimes [19].

Examination of each term in the momentum equation [Eq. (3)] reveals that all assumptions made in the Sweet-Parker model hold true, except that $p_{\text {down }} \gg p_{\text {up }}$ (dominated by differences in density), as measured by spatial scans of a Langmuir probe. Thus the momentum equation is modified to $V_{Z}=\sqrt{V_{A}^{2}-2\left(p_{\text {down }}-p_{\text {up }}\right) / \rho}$, where the outflow is lessened by the higher $p_{\text {down }}$.

As a result, the reconnection rate $M_{A}$ is modified to $\left(1 / \sqrt{S^{*}}\right) \sqrt{\left(1+\operatorname{Ln} / n V_{Z}\right)\left(V_{Z} / V_{A}\right)} \equiv$ $1 / \sqrt{S_{\text {eff }}}$, where the effective Lundquist number is defined as $S_{\text {eff }}=\left(\mu_{0} L V_{A} / \eta^{*}\right) /[(1+$ $\left.\left.L \dot{n} / n V_{Z}\right)\left(V_{Z} / V_{A}\right)\right]$. The effects of compressibility and downstream pressure are shown in Fig. 5, where the terms $L \dot{n} /\left(n V_{Z}\right)$ and $V_{Z} / V_{A}$ are plotted as functions of $M_{A}$. As $S$ increases, the reconnection rate decreases and the outflow is slower due to the downstream pressure. At the same time, the effect of compressibility becomes increasingly important in a narrower current sheet. Compressibility, which allows local density build-up, explains why higher central density is observed in discharges with higher field even though the initial density is the same.

The observed reconnection rate is plotted against $1 / \sqrt{S_{\text {eff }}}$ in Fig. 6. As expected, they are in good agreement. The classical Sweet-Parker model needs to be generalized to incorporate the compressibility, the downstream pressure, and the effective resistivity. Effects of the compressibility must be transient (as in MRX) since the density accumulation cannot be sustained indefinitely. However, occurrences of magnetic reconnection in nature do not have 
to be steady state. They can be impulsive locally while global structures are maintained in a quasi (slowly evolving) steady state, as supported by a recent computer simulation using compressible MHD equations [20].

The effect of downstream pressure is easy to understand. As observed in MRX, higher plasma pressure in the downstream region slows the outflow, thus reducing the reconnection rate. One can envision another case in which lower pressure in the downstream region can result in super-Alfvénic outflow, leading to an increase in the reconnection rate, a situation which can exist in solar flares and other cosmic environments.

One relevant question might be whether Petschek-type models can explain the same observations. Direct comparisons, however, are not possible since these shock-based models do not predict definite reconnection rates, only their maxima [2]. Shock structures, which are an essential feature of the Petschek-type models, would appear as multiple jumps in $B_{Z}(R)$ profiles in the downstream region if they existed. However, these jumps have not yet been observed within the sensitivity limits of the measurements in MRX.

In summary we have performed for the first time, to the best of our knowledge, an experimental test of the Sweet-Parker model of magnetic reconnection in a laboratory plasma. It is found that the observed reconnection rate cannot be explained by the Sweet-Parker model unless the model is generalized to incorporate compressibility, downstream pressure, and the effective resistivity. The latter is significantly enhanced over its classical values in the collisionless limit. Then a main question is how the resistivity is enhanced over its classical value in the collisionless regime. It is not surprising if current-carrying electrons are scattered by micro-instabilities destabilized in the diffusion region, where high current density (therefore large drift parameter) and large inhomogeneity (in plasma pressure and magnetic field) exist. In fact, the drift parameter, $v_{\mathrm{d}} / v_{\text {the }}\left(v_{\mathrm{d}}=j_{T} / e n\right.$ and $v_{\text {the }}=$ electron thermal velocity), is found to be a constant $\sim 0.1$ in MRX, independent of the reconnection rate. This suggests that instabilities driven by relative drift between ions and electrons provide a mechanism to limit current density, thus controlling the reconnection rate. There is evidence that impurity content can also affect the reconnection process through energy transport, as 
suggested by observations of lower $T_{e}$ in the diffusion regions of dirty plasmas. However, a complete self-consistent picture for the resistivity enhancement requires fine scale, full 3D dynamic measurements, which will be a subject of further pursuit in MRX.

The authors are grateful to K. Morrison, D. Cylinder, and T. Carter for their technical contributions. This work was jointly supported by NASA, NSF, ONR, and DOE. 


\section{REFERENCES}

[1] E. N. Parker, in Cosmical Magnetic Fields (Clarendon Press, Oxford, 1979).

[2] V. M. Vasyliunas, Rev. Geophys. Space Phys. 13, 303 (1975).

[3] D. Biskamp, Physics Report 237, 179 (1993).

[4] See, for example, Introduction to Space Physics (Ed. by M.G. Kivelson and C.T. Russell, Cambridge, 1995) and references therein.

[5] For example, J.B. Taylor, Rev. Mod. Phys. 58, 741(1986).

[6] R. G. Giovanelli, Nature 158, 81 (1946).

[7] P. A. Sweet, in Electromagnetic Phenomena in Cosmical Physics, ed. B. Lehnert (Cambridge University Press, New York, 1958), pp.123.

[8] E. N. Parker, J. Geophys. Res. 62, 509 (1957).

[9] H. E. Petschek, NASA Spec. Pub. SP-50, 425 (1964).

[10] B. U. Ö. Sonnerup, J. Plasma Phys. 4, 161 (1970).

[11] E. R. Priest and T. G. Forbes, J. Geophys. Res. 91, 5579 (1986).

[12] See, for example, J. F. Drake et al., Phys. Rev. Lett. 73, 1251 (1994) and references therein.

[13] R. L. Stenzel and W. Gekelman, J. Geophys. Res. 86, 649 (1981).

[14] M. Yamada et al., Phys. Rev. Lett. 65, 721 (1990); Y. Ono et al., Phys. Rev. Lett. 76, 3328 (1996).

[15] M. Yamada et al., Phys. Rev. Lett. 78, 3117 (1997).

[16] M. Yamada et al., Phys. Plasmas 4, 1936 (1997).

[17] E. G. Harris, Nuovo Cimento 23, 115 (1962). 
[18] R. M. Kulsrud, to be published; S. I. Braginskii, in Rev. of Plasma Physics 1 (Consultants Bureau, New York, 1966).

[19] In discharges with high $p_{\text {fill }}(\gtrsim 10 \mathrm{~m}$ Torr), the source term appears to be non-negligible; this would lead to a reduced inflow in the continuity equation.

[20] H. Kitabata et al., J. Phys. Soc. Jap. 65, 3208 (1996). 


\section{FIGURES}

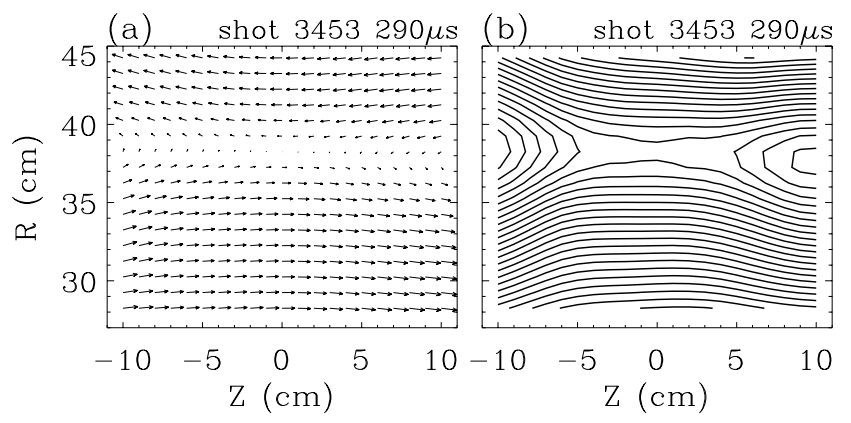

FIG. 1. An example of driven magnetic reconnection measured in a single shot by a 2D probe array in MRX: (a) vector plot of poloidal field, (b) poloidal flux contours. Toroidal field (the third component) is negligibly small.

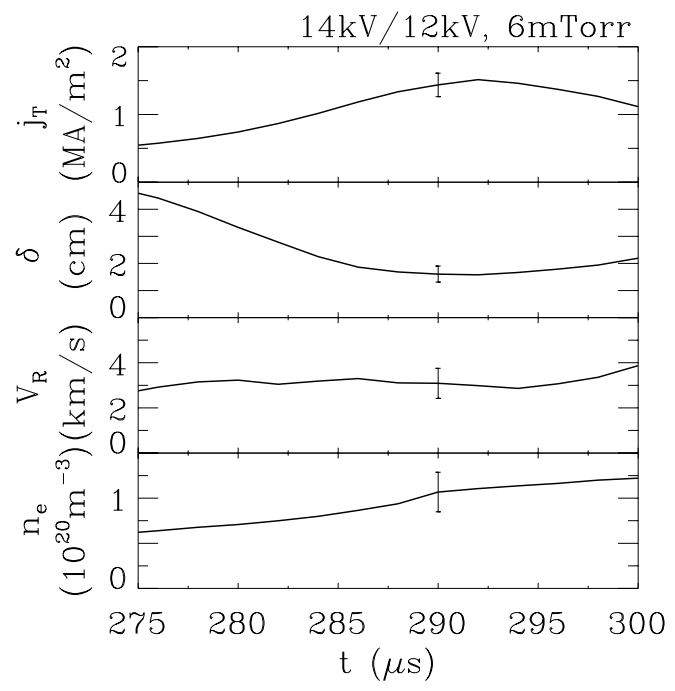

FIG. 2. A shot-averaged time evolution of driven magnetic reconnection. From top: peak current density, current sheet thickness, inflow speed at $R=30 \mathrm{~cm}$ from flux movement, electron density at center. 

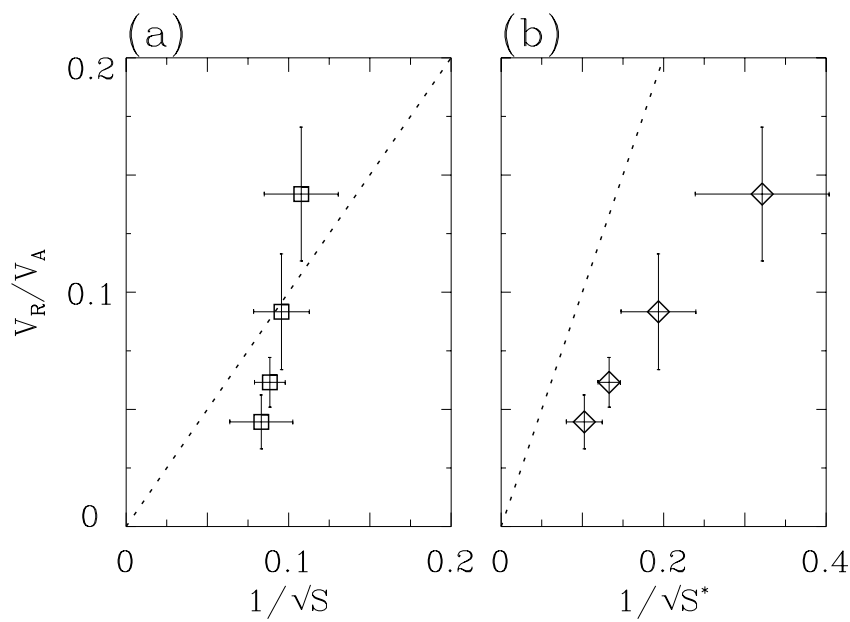

FIG. 3. Experimental test of the Sweet-Parker model (represented by the dotted lines): reconnection rate vs. (a) $1 / \sqrt{S}$ and (b) $1 / \sqrt{S^{*}}$, where $S$ and $S^{*}$ are calculated from the classical and measured resistivity, respectively.

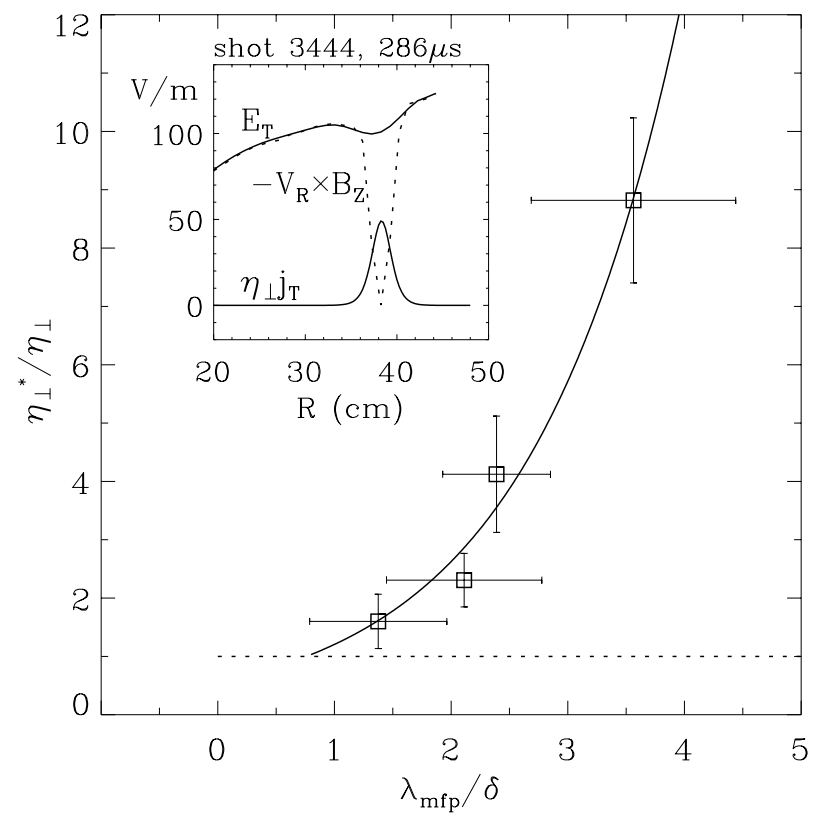

FIG. 4. Resistivity enhancement as a function of collisionality characterized by the ratio of electron mean free path to current sheet thickness. An example of all three terms of Ohm's law across the current sheet is shown in the inset. 


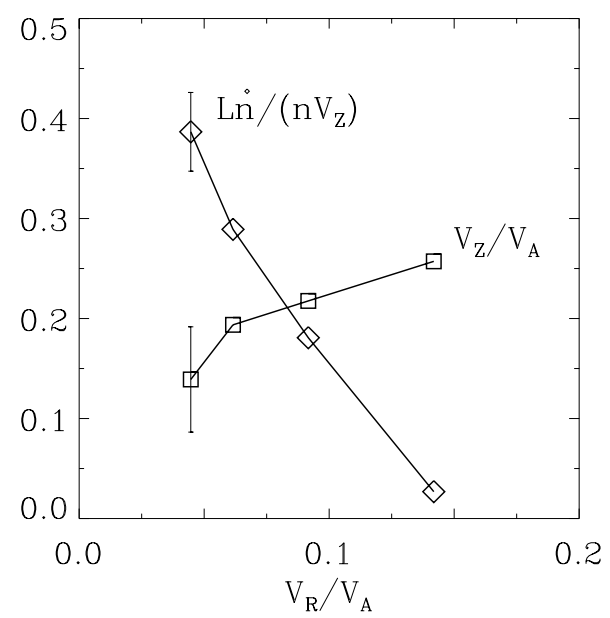

FIG. 5. Effects of compressibility (open diamonds) and downstream pressure (open squares) as functions of reconnection rate. As reconnection rates decreases, the outflow is further slowed by downstream pressure while the effect of compressibility becomes increasingly important.

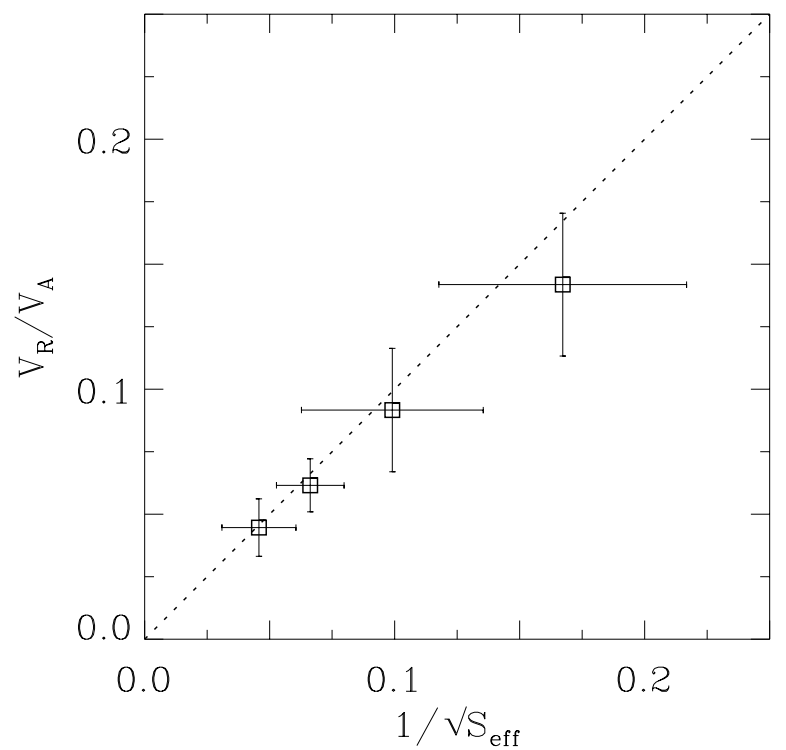

FIG. 6. The observed reconnection rates are compared to the prediction by a generalized Sweet-Parker model, $1 / \sqrt{S_{\text {eff }}}$, which incorporates finite compressibility, downstream pressure and the effective resistivity. 